Kai A. Konrad

Marcel Thum

\title{
Does a Clean Development Mechanism Facilitate International Environmental Agreements?
}

\author{
Max Planck Institute for Tax Law and Public Finance \\ Working Paper 2014 - 20
}

November 2014

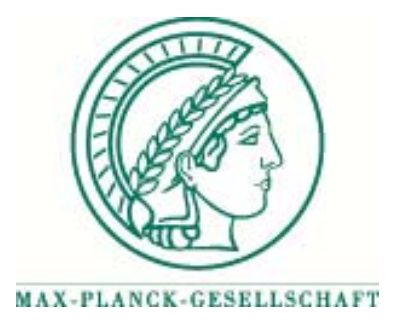

Max Planck Institute for

Tax Law and Public Finance

Department of Business and Tax Law

Department of Public Economics

http://www.tax.mpg.de 
Working papers of the Max Planck Institute for Tax Law and Public Finance Research Paper Series serve to disseminate the research results of work in progress prior to publication to encourage the exchange of ideas and academic debate. Inclusion of a paper in the Research Paper Series does not constitute publication and should not limit publication in any other venue. The preprints published by the Max Planck Institute for Tax Law and Public Finance represent the views of the respective author(s) and not of the Institute as a whole. Copyright remains with the author(s).

Max Planck Institute for Tax Law and Public Finance

Marstallplatz 1

D-80539 Munich

Tel: $\quad+498924246-0$

Fax: $\quad+498924246-501$

E-mail: ssrn@tax.mpg.de

http://www.tax.mpg.de 


\title{
Does a Clean Development Mechanism Facilitate International Environmental Agreements?
}

\author{
Kai A. Konrad* and Marcel Thum ${ }^{\dagger}$
}

August 27, 2014

\begin{abstract}
When politicians negotiate in international climate conventions they may suffer from incomplete information about each other's preferences about reaching an agreement. As is known, this may cause failure to reach an efficient cooperative agreement. We study the role of the clean development mechanism (CDM) for the likelihood of such failure. The CDM has been introduced in the context of the Kyoto Protokol to allow countries to make efficiency enhancing use of cross-country lowcost mitigation opportunities. We use a simple bargaining framework to uncover why this mechanism may reduce the likelihood for reaching an efficient cooperative climate agreement.

JEL classification code: Q54, Q58, F53, H41

Keywords: clean development mechanism, international climate agreements, bargaining, incomplete information
\end{abstract}

\footnotetext{
* Max Planck Institute for Tax Law and Public Finance, Munich, Germany.

${ }^{\dagger}$ Faculty of Business and Economics, TU Dresden, and ifo Dresden, Germany.
} 


\section{Introduction}

Article 12 of the Kyoto protocol introduced the principle that signatory states can fulfill part of their commitments towards greenhouse gas reductions by implementing abatement projects in developing countries that have ratified the protocol but have made no commitment. ${ }^{1}$ The objectives of this Clean Development Mechanism (CDM) were twofold: On the one hand, developing countries should benefit from technology transfers and achieve a sustainable development. On the other hand, the industrialized countries should be able to minimize their abatement costs. It is this latter objective of the CDM our paper focuses on. The industrialized countries will benefit if some abatement projects can be carried out at lower costs in high polluting industries abroad. Due to the lower cost, the industrialized countries may even find it optimal to increase their commitment towards abatement compared to a scenario without CDM. In this paper we identify a major potential drawback of the CDM: The availability to the CDM changes the cost and benefit of a cooperation agreement in climate negotiations. As it turns out, the lower benefits will lead to more aggressive bargaining and this will ultimately reduce the probability for an international agreement on climate policy.

We employ a formal bargaining framework with two countries, or two groups of countries if these groups coordinate internally and bargain as single strategic players. We consider ultimatum bargaining. One country is the proposer, offers an emission reduction of a given size to the other country and asks for a transfer in compensation. The other country is the responder and may accept or reject. As is well-known, ultimatum bargaining may fail to reach an efficient outcome if the proposer has incomplete information about relevant aspects of the responder's payoff function. In this context politicians represent their countries and take the roles as negotiators in the climate conventions. Politicians may have their own objective functions. They may care more or less strongly about negotiation success. And politicians can be imperfectly informed about their negotiation partner's political benefits or costs of reaching an environmental treaty. This may lead to a failure to reach a cooperative agreement, even if such an agreement has a positive aggregate surplus. The proposer, being uncertain about parameters of the objective function of the responder politician, may offer too little emission reduction and/or ask for a too large compensation. Hence, the model allows for the possibility of failure in negotiations due to this incomplete information about

\footnotetext{
${ }^{1}$ For a comprehensive survey on the CDM literature, see Paulsson (2009).
} 
the negotiating politicians' preferences. $^{2}$

The risk of failure in negotiations depends on the specific framework and parameters. We compare two frameworks that differ in the fallback that is reached if negotiations fail. In one framework a CDM is available only as part of the cooperative agreement. A CDM is unavailable in the non-cooperative fallback that is reached if bargaining fails. Hence, if the negotiations fail, the domestic firms have to fulfil their obligations and can rely only on domestic abatement projects. In the second framework the countries have agreed already on a CDM that applies even in the case of negotiation failure; even when negotiations fail, the domestic firms in the proposer country can make use of abatement projects in the responder country. The two frameworks differ in the aggregate benefits of reaching an agreement. With a CDM in place, the offer-making country will find it optimal to choose a higher abatement level - even when negotiations fail. This reduces the aggregate welfare gains from negotiations compared to the framework that rules out the use of CDM in case of negotiation failure. The smaller potential welfare gain makes negotiation failure less painful and this gives rise to a more agressive bargaining behavior of the proposer country. To maximize its own expected payoff, the proposer will ask for a higher transfer in exchange for additional abatement. This aggressive bargaining behavior increases the likelihood for a negotiation failure.

Ultimatum bargaining under incomplete information is a simple and prominent tool that can be used to reveal, assess and compare the possibility for bargaining to reach an inefficient outcome in a context of incomplete information. As is known from Myerson and Satterthwaite (1983) and Chatterjee and Samuelson (1983), the inefficiency in the context of incomplete information is generic, and not due to the specific type of bargaining set-up. ${ }^{3}$

There is also an extensive literature on the role of transfers in inter-

\footnotetext{
${ }^{2}$ This is in contrast to models of cooperative Nash bargaining or non-cooperative bargaining under complete information where negotiations can never fail. Models that employ Nash bargaining in the context of abatement consider the role of unilateral commitment on abatement (Hoel 1991), commitment on technology choices (Buchholz and Konrad 1994), or investment in R\&D (Beccherle and Tirole 2011), prior to bargaining.

${ }^{3} \mathrm{~A}$ different approach on negotiations that can be applied to climate negotiations is by Martimort and Sand-Zantman (2013) and Helm and Wirl (2014). They consider a richer contractual framework. Such a richer contractual framework may, but need not exist. If, as in Helm and Wirl (2014), one side can commit and offer a whole menu of price-quantity contracts, then our research question would have to be redefined. It would no longer be a question on whether or not a cooperative agreement is found, but the question would be how the efficiency properties of the principal-agent contract that is reached in the equilibrium depends on the availability of a CDM in case of non-cooperation.
} 
national environmental agreements. This literature mostly focuses on the question whether side payments can increase the set of signatory countries (see, for instance, Barrett (2001) or Carraro and Siniscalco (1993)). Or it discusses the feedback effects as the anticipation of future transfers might reduce the incentives to commit to cooperation in the first place; see Hoel and Schneider (1997). These papers, however, do not consider the potential failure of international environmental negotiations.

With respect to CDM, there is an extensive literature on whether the proposed objectives are achieved (see Paulsson 2009). In particular, the question of additionality has received quite some attention in the literature. These considerations are largely orthogonal to the question of whether or not a CDM makes bargaining failure more or less likely. ${ }^{4}$ From a technical point of view, our paper is closely related to Harstad (2007) as well as Konrad and Thum (2014). Both papers allow for negotiation failures. Harstad shows that side payments can be detrimental to successful bargaining. In Konrad and Thum (2014), we analyse the role of unilateral commitment on advances in greenhouse-gas abatement for the likelihood of successful negotiations, also using ultimatum offer bargaining. The ultimatum bargaining process is admittedly simple, but it highlights the economic rationale behind failed negotiations in the context of incomplete information. As described in Barrett (1998), climate negotiations are a much more complex matter that has further dynamics, other important elements and relevant dimensions. However, the simple bargaining framework applied here reveals a strong intuition which should also be relevant in more complex frameworks.

\section{The formal analysis}

Overview In short, we consider a world with two countries $A$ and $B$ that suffer from global warming, derive benefits from global greenhouse gas emission reduction and negotiate about a global agreement. We may think of $A$ as a rich country (or the industrialized hemisphere) with few unexploited cost-effective emission reduction opportunities at home, and of $B$ as a less

\footnotetext{
${ }^{4}$ Abatement projects that are carried out within the CDM are desired to be additional to what the host country would have undertaken anyhow. Additionality is hard to verify as the counterfactual is not observable. To focus on the CDM-bargaining nexus, we eliminate this aspect by assuming that only the Annex 1 countries abate in laissez-faire. Aresin (2013) focuses on the strategic interaction between countries. Her analysis is constrained to a fully non-cooperative framework. She analyses the welfare effects of the CDM, depending on whether low cost abatement opportunities in the Annex 2 countries are allocated to firms from this country.
} 
wealthy country (or less developed hemisphere) that provides considerable opportunity for cost effective emission reductions. Politicians who represent these countries appear at the negotiation table of a climate convention. With a slight abuse of notation, we denote them as politicians $A$ and $B$. In the negotiations for a climate agreement, $A$ is the proposer and $B$ is the responder in an ultimatum offer game. The proposer will offer the responder a given emission reduction program and will ask for/ offer a transfer. If the negotiations lead to an environmental agreement, this agreement will be implemented. If negotiations fail, each country will non-cooperatively implement the country's own non-cooperative optimum. A crucial aspect for this choice is whether $A$ can mitigate only using mitigation opportunities in country $A$ or whether there is a CDM in operation that offers less costly mitigation opportunties to $A$ in country $B$, even if negotiations about a climate convention fail. We compare the games with and without a CDM with respect to the likelihood by which a global climate agreement can be reached.

To address this, we first describe the mitigation technology and the role of a CDM for mitigation opportunities and costs before we describe the benefits from mitigation for the countries. Then we consider the agenda of the politicians who act as proposers and responders. Next we characterize the cooperative outcome and the non-cooperative outcomes that emerge if bargaining fails to reach an agreement. Finally we turn to the choices in the bargaining game, derive the optimal strategies and the equilibrium and compare the probabilities for bargaining success with and without a CDM.

Mitigation technology First we characterize the technological opportunities for climate preserving emission mitigation and their costs. For simplicity, let the industry with climate relevant emissions be the firms in country $A$, and let $A$ be the country that can regulate the mitigation of these firms. Assuming that all emission decisions are made in $A$ disregards the possible regulatory/polution problems that may emerge in country $B$. This is a useful simplification as it removes the problem of additionality that may otherwise occur in the context of a CDM. The assumption allows us to focus on the bargaining inefficiency. The choice variable is emission reduction. Such emission reduction can occur domestically in $A$, making use of mitigation opportunities in country $A$, or in country $B$. The quantities of emission reduction that use mitigation opportunities in $A$ and $B$ are denoted by $m_{A}$ and $m_{B}$ and they sum up to total global emission reductions $m=m_{A}+m_{B}$. Emission reductions in country $A$ have a cost $c_{A}\left(m_{A}\right)$ 
and mitigation that is carried out in country $B$ has a cost $c_{B}\left(m_{B}\right)$. These cost functions are twice continuously differentiable and convex, with $c_{i}^{\prime}>0$, $c_{i}^{\prime \prime}>0$ and $c_{i}(0)=c_{i}^{\prime}(0)=0$ for $i \in\{A, B\}$.

Note that the total cost of aggregate mitigation $m$ that is implemented by the government of country $A$ depends on the use of mitigation opportunities in $A$ and $B$. In the absence of a CDM, firms in country $A$ can make use of mitigation opportunities in country $A$ only. Accordingly, $m_{A}=m$, and the mitigation cost is $c_{A}(m)$.

If a CDM is available, then the firms can make use of the mitigation opportunities in country $B$, too. A cost-minimizing mitigation program with aggregate mitigation $m=m_{A}+m_{B}$ uses mitigation opportunities in both countries as a solution of

$$
\min _{m_{A}}\left\{c_{A}\left(m_{A}\right)+c_{B}\left(m-m_{A}\right)\right\} \equiv c(m) .
$$

For any given aggregate amount of mitigation, $c(m)$ is the cost as a function of $m$. A key assumption in what follows is that

$$
c(m)<c_{A}(m) \text { for all } m>0 .
$$

This is a mild assumption. It basically states that the availability of mitigation opportunities in $B$ is economically relevant. If Assumption (M) does not hold, the CDM is essentially meaningless in this economy and the question we address also becomes irrelevant. If Assumption (M) holds, then the use of a CDM reduces total mitigation costs compared to using mitigation opportunities only in country $A$.

Mitigation benefits Next we consider countries' payoffs. Global mitigation affects climate change, but the geographical origin of emission reductions does not matter for the impact of greenhouse gas emissions. Accordingly, countries have benefits from global mitigation $m$. The countries' benefits from mitigation $m$ are functions $b_{A}(m)$ and $b_{B}(m)$. These gross benefits can be understood as the aggregate rent emerging in the respective country if the aggregate mitigation is $m$, notwithstanding the cost of mitigation. For welfare comparisons these benefits will be compared with the mitigation costs. ${ }^{5}$ The two countries may have the same or different benefit functions, but we assume that these functions are both twice continuously differentiable and concave, with $b_{i}^{\prime}>0$ and $b_{i}^{\prime \prime}<0$ for $i \in\{A, B\}$ with $b_{i}^{\prime}(0)$ being sufficiently large.

\footnotetext{
${ }^{5}$ Benefits of mitigation can be expected to be unevenly distributed in a country, but we disregard such intra-country equity issues and assume that the benefits can be uniquely aggregated and represented by these functions.
} 
Politicians' agenda - the source of incomplete information We assume that all cost and benefit functions introduced so far are common knowledge. This may be reasonable, given that mitigation technology is not top secret and also what can be found out about the mitigation benefits in one country relies on sources that are typically openly accessible, at least to experts and specialists. However, in addition to these technologically determined costs and benefits, the politician who negotiates for country $B$ may receive a political benefit or cost of size $\beta$ if bargaining leads to a global environmental agreement, compared to bargaining failure. For instance, successful negotiations may be considered an indicator for the politician's general ability, may be interpreted as a diplomatic success and may help being reelected or gaining support from environmental groups at home. The size of this political benefit $\beta$ cannot be observed so easily. It is the politician's private information. It may be large and positive, but it may as well be small or even negative. This may be true if the politician cares particularly about voters who fear job losses from additional mitigation via the CDM, or cares about the support from an industry lobby that suffers from an implementation of high mitigation effort. ${ }^{6}$

From the point of view of country $A$, the political benefits $\beta$ are not observable. The politician $B$ knows his own $\beta$, but the politicians in country $A$ making the offer to $B$ only know the distribution from which these political benefits are a random draw. This incomplete information is a possible source of bargaining failure. The political benfits are distributed on the interval $\beta \in[\beta, \bar{\beta}]$, where $\beta$ may be negative. Let $F(\beta)$ denote the cumulative distribution function from which $\beta$ is drawn, with $F^{\prime}(\beta) \geq 0$. Throughout we also assume that the hazard rate is non-increasing in $\beta$, i.e.,

$$
\frac{d}{d \beta}\left([1-F(\beta)] / F^{\prime}(\beta)\right) \leq 0 .
$$

Assumption ( $\mathrm{H})$ holds for many commonly considered probability distributions and is a standard regularity assumption in contract theory.

Non-cooperative and cooperative mitigation outcomes As discussed before, we distinguish two frameworks that differ with respect to the avail-

\footnotetext{
${ }^{6}$ It is important for the problem we study that the proposer is uninformed. Politician $A$, who is the proposer in our framework, may actually have an own private political benefit or cost from negotiation failure as well, and this benefit may be $A$ 's private information. Generalizing our framework accordingly would not qualitatively affect our results. Of course, the prevalence of mutual incomplete information justifies our framework, as it explains why it would not solve the problem of bargaining failure if $A$ and $B$ switched their roles as proposer and responder.
} 
ability of a CDM in case the bargaining procedure does not yield an environmental agreement.

Consider first the framework in which country $A$ cannot use the CDM in country $B$ if negotiations fail. In this case country $A$ chooses the mitigation level that is optimal in autarky, i.e. considering only domestic mitigation projects with marginal costs $c_{A}^{\prime}(m)$. The individually optimal choice of $m=m_{A}$ in this case is the solution of

$$
c_{A}^{\prime}(m)=b_{A}^{\prime}(m)
$$

Consider next the case with a CDM being in place even if bargaining fails to reach an agreement. This second framework allows country $A$ 's firms to use the mitigation opportunities in country $B$. The cost of a given amount of abatement $m=m_{A}+m_{B}$ is described by the cost function $c(m)$. As a result, the non-cooperative fallback which is chosen by country $A$ in the case of negotiation failure is described by the solution of

$$
c^{\prime}(m)=b_{A}^{\prime}(m)
$$

Finally, consider the efficient amount of $m$ that emerges if both countries' benefits of of abatement $m$ are taken into account. Efficiency also implies that the low-cost abatement opportunities in country $B$ are used. Accordingly, the efficient amount of abatement is determined by the condition

$$
c^{\prime}(m)=b_{A}^{\prime}(m)+b_{B}^{\prime}(m) .
$$

The choice of $m$ and the availability of a CDM determines the environmental cost and benefits from mitigation for the two countries. Recall that, given the availability of information about technologies and their costs, the functions $c_{A}(m), c(m), b_{A}(m)$ and $b_{B}(m)$ are common knowledge. This makes also the solutions to (1), (2) and (3) common knowledge. We denote the solutions to these equations as $m_{1}, m_{2}$ and $m_{3}$. And, as is clear from these marginal conditions, the assumptions about the cost and benefit functions and the assumption about the economic relevance of the CDM, we have

$$
m_{3}>m_{2}>m_{1} \text {. }
$$

Intuitively, accounting only for country $A$ 's benefits, the emission reduction that is optimal for $A$ is higher if - due to the CDM - the cost of emission reduction is lower: $m_{2}>m_{1}$. Given these lower costs, optimal emission reduction is even higher if one accounts for both countries' benefits. 
Countries' rents in the three outcomes These mitigation levels lead to different mitigation benefits $b_{i}$ in countries $i=A, B$, and to mitigation costs in country $A$. We can determine the net benefits that emerge in the two countries for each of the three outcomes in the absence of transfers between the two countries. These are

$$
\begin{aligned}
b_{A}\left(m_{1}\right)-c_{A}\left(m_{1}\right) & \equiv \theta_{1} \\
b_{A}\left(m_{2}\right)-c\left(m_{2}\right) & \equiv \theta_{2} \\
b_{A}\left(m_{3}\right)-c\left(m_{3}\right) & \equiv \theta_{3},
\end{aligned}
$$

and the net benefits for country $B$ are equal to their gross benefits $b_{B}\left(m_{1}\right)$, $b_{B}\left(m_{2}\right)$ and $b_{B}\left(m_{3}\right)$. The following properties are straightforward to show: First,

$$
\theta_{1}<\theta_{2} \text { and } b_{B}\left(m_{1}\right)<b_{B}\left(m_{2}\right) .
$$

If the non-cooperative outcome emerges, both countries are better-off if a CDM is in place. Second,

$$
\theta_{3}<\theta_{2} \text { but } \theta_{3}+b_{B}\left(m_{3}\right)>\theta_{2}+b_{B}\left(m_{2}\right) .
$$

Essentially, country $A$ prefers outcome $m_{2}$ to $m_{3}$ in the absence of transfers, but the sum of rents in both countries is higher for $m_{3}$ than for $m_{2}$. This follows by construction, as $m_{3}$ maximizes this sum for all possible $m$.

Ultimatum bargaining Country $A$ implements $m$ and bears the technological cost of this mitigation. Country $A$ also has some of the benefits of emission abatement, but some part of the benefits of emission abatement are generated using abatement opportunities in country $B$. In a non-cooperative solution, country $A$ chooses $m$ in order to maximize own payoff, not taking into account that $B$ has a willingness to pay for an increase in emission abatement $m$. For these external effects, the two countries can bargain over the total amount of emissions. We consider ultimatum bargaining. The proposer country/politician $A$ can make a take-it-or-leave-it offer to the responder $B$. This offer states the total emission reduction $m$ that $A$ will implement through regulation of its domestic firms and asks for a transfer $t$ payment from country $B$ to country $A$. The responder $B$ decides about whether to accept or reject this offer. If $B$ rejects, then country $A$ implements the domestically optimal mitigation level. If $B$ accepts, then $m$ is implemented and $B$ pays $t$ to $A$. 
Bargaining over mitigation So far we have characterized three possible mitigation outcomes as well as the mitigation costs and mitigation benefits for countries $A$ and $B$ that emerge in these outcomes. Now we turn to a possible ultimatum bargaining game that makes use of these three outcomes. Proposer/politician $A$ makes a take-it-or-leave-it offer to responder/politician $B$. The offer states the amount $m_{3}$ of mitigation country $A$ is willing to impose on its firms and a transfer $t$ that is paid to country $A$ by country $B$. Country $B$ needs to decide whether to accept this offer or to reject it. Note that the restriction to offers with $m=m_{3}$ limits the set of possible offers that can be made to the set of efficient outcomes. ${ }^{7}$

If the offer is accepted, then $m_{3}$ is implemented and country $B$ pays $t$ to country $A$. If the offer is rejected, then this ends the bargaining stage. Country $A$ then chooses the amount of mitigation that maximize $A$ 's rents and no transfers are paid. This amount of mitigation is $m_{A}=m=m_{1}$ if no CDM is available in the non-cooperative fallback, and it is $m_{A}+m_{B}=m_{2}$ if a CDM is available in the non-cooperative fallback. Hence, the fallback of ultimatum bargaining is the non-cooperative equilibrium without or with a CDM, respectively.

Turn now to $A$ 's offer. Country $A$ offers the efficient amount of mitigation $m_{3}$ in any case, but $A$ chooses the transfers $t$ demanded, and this transfer depends on the fallback position. We discuss the two alternatives - a fallback without a CDM and a fallback with a CDM - in turn.

Case 1: no CDM is available in the fallback A successful climate agreement changes mitigation from $m_{1}$ to $m_{3}$, net benefits for the proposer $A$ from $\theta_{1}$ to $\theta_{3}+t$ and benefits for the politician responder from $b_{B}\left(m_{1}\right)$ to $b_{B}\left(m_{3}\right)+\beta-t$. Responder $B$ accepts the offer if $b_{B}\left(m_{3}\right)-b_{B}\left(m_{1}\right)-t+\beta \geq 0$, or, solving for $\beta$, if

$$
\beta \geq b_{B}\left(m_{1}\right)-b_{B}\left(m_{3}\right)+t .
$$

This inequality describes a one-to-one relationship between $t$ and the critical $\beta$ such that $B$ accepts the transfer demanded if the transfer is at most equal

\footnotetext{
${ }^{7}$ The limitation to a fixed $m$ is important as only this limitation allows us to study bargaining failure and whether a CDM increases or decreases its likelihood. A more sophisticated bargaining framework in the spirit of Martimort and Sand-Zantman (2013) or Helm and Wirl (2014) could allow $A$ to offer a menu of possible contracts $(m, t)$. Player $A$ could then typically implement the optimal principal-agent contract. In this case, rather than inefficiency due to failure to sign a contract, an inefficiency with respect to the size of $m$ emerges for contracts with all agent types, except 'at the top'. A question that is analogous to our question could be studied within a principal-agent framework: whether a CDM in the default state increases or decreases the expected inefficiency that emerges in the optimal principal-agent contract.
} 
to the $t$ that solves this relationship with equality. We denote this critical $\beta$ as a function of $t$ for the case without a CDM available in the fallback as

$$
\beta_{1}(t)=b_{B}\left(m_{1}\right)-b_{B}\left(m_{3}\right)+t .
$$

This function is linear in $t$ and has slope 1 .

Inequality (5) yields a bargaining-success probability of $1-F\left(b_{B}\left(m_{1}\right)-\right.$ $\left.b_{B}\left(m_{3}\right)+t\right)$. The proposer $A$ chooses $t$ to maximize

$$
E \pi_{A, 1}=\left(\theta_{3}-\theta_{1}+t\right)\left(1-F\left(b_{B}\left(m_{1}\right)-b_{B}\left(m_{3}\right)+t\right)\right) .
$$

This expected payoff is the product of the actual gain in case of success compared to the fallback, times the probability for acceptance of the proposal. Differentiating with respect to $t$ yields a first-order condition

$$
\frac{\partial E\left(\pi_{A, 1}\right)}{\partial t}=0
$$

which can be re-written as

$$
\frac{1-F\left(b_{B}\left(m_{1}\right)-b_{B}\left(m_{3}\right)+t_{1}\right)}{F^{\prime}\left(b_{B}\left(m_{1}\right)-b_{B}\left(m_{3}\right)+t_{1}\right)}=\theta_{3}-\theta_{1}+t_{1},
$$

where $t_{1}$ denotes the solution of this equation where it exists. Note that the left-hand side of (6) is weakly decreasing in the transfer demanded (due to Assumption $(\mathrm{H})$ of a non-increasing hazard rate), and the right-hand side is strictly monotonically increasing in the transfer. Accordingly, there is at most one transfer level $t$ that fulfills this first-order condition. We denote this solution by $t_{1}$.

Case 2: a CDM is available in the fallback A successful climate agreement changes mitigation from $m_{2}$ to $m_{3}$, net benefits for the proposer $A$ from $\theta_{2}$ to $\theta_{3}+t$ and benefits for the responder from $b_{B}\left(m_{2}\right)$ to $b_{B}\left(m_{3}\right)-t+\beta$. Responder $B$ accepts the offer if $b_{B}\left(m_{3}\right)-b_{B}\left(m_{2}\right)-t+\beta \geq 0$, or, solving for $\beta$, if

$$
\beta \geq b_{B}\left(m_{2}\right)-b_{B}\left(m_{3}\right)+t .
$$

This inequality describes a one-to-one relationship between $t$ and the critical $\beta$ such that $B$ accepts the transfer offer if the transfer is at least equal to the $t$ that solves this relationship with equality. We denote this critical $\beta$ as a function of $t$ for the case with a CDM available in the fallback as

$$
\beta_{2}(t)=b_{B}\left(m_{2}\right)-b_{B}\left(m_{3}\right)+t .
$$


This function is linear in $t$ and has slope 1 .

The relationship (7) yields a success probability of $1-F\left(b_{B}\left(m_{2}\right)-\right.$ $\left.b_{B}\left(m_{3}\right)+t\right)$. The proposer $A$ chooses $t$ to maximize

$$
E \pi_{A, 2}=\left(\theta_{3}-\theta_{2}+t\right)\left(1-F\left(b_{B}\left(m_{2}\right)-b_{B}\left(m_{3}\right)+t\right)\right) .
$$

This expected payoff is the product of the actual gain in case of success, compared to the fallback, times the probability for acceptance of the proposal. Differentiating with respect to $t$ yields a first-order condition which can be rewritten as

$$
\frac{1-F\left(b_{B}\left(m_{2}\right)-b_{B}\left(m_{3}\right)+t_{2}\right)}{F^{\prime}\left(b_{B}\left(m_{2}\right)-b_{B}\left(m_{3}\right)+t_{2}\right)}=\theta_{3}-\theta_{2}+t_{2} .
$$

Note that the left-hand side of (6) is again weakly decreasing in the transfer (due to Assumption $\mathrm{H}$ of a non-increasing hazard rate), and the right-hand side is strictly monotonically increasing in the transfer. Accordingly, there is at most one $t$ that fulfills this first-order condition. We denote this solution by $t_{2}$.

The correspondence between $t$ and $\beta$ We observe a one-to-one correspondence between $t$ and a value of $\beta(t)$ such that responder $B$ accepts the transfer offer $t$ if $\beta>\beta(t)$ and rejects the transfer offer if $B$ 's true value of $\beta$ is smaller than $\beta(t)$ in the interior range of $(\beta, \bar{\beta})$. With a mild abuse of notation, let $\beta_{k}$ denote the political benefit necessary to make country $B$ indifferent between accepting and rejecting the offer $t_{k}$ in case $k(k=1,2)$. As shown by (5) and (7), the respective functions $\beta_{k}(t)$ are linear and have slope 1 for $\beta_{k}(t) \in(\beta, \bar{\beta})$ and a zero slope outside this interval. Further, $\beta_{2}(t)-\beta_{1}(t)=b_{B}\left(m_{2}\right)-b_{B}\left(m_{1}\right)>0$.

A comparison of bargaining success probabilities Suppose interior solutions $t_{1}$ and $t_{2}$ exist. We can compare the likelihood for a coopperative outcome in this case.

Proposition 1 Let $t_{1}$ and $t_{2}$ describe A's demands in cases 1 and 2 (that is, without and with a CDM in the non-cooperative fallback). Then it holds that the probability for a bargaining failure is higher if a CDM is available in the non-cooperative fallback.

Proof. Note that $t_{1}=\beta_{1}+b_{B}\left(m_{3}\right)-b_{B}\left(m_{1}\right)$ and $t_{2}=\beta_{2}+b_{B}\left(m_{3}\right)-b_{B}\left(m_{2}\right)$. Then we can rewrite the first-order conditions as

$$
\frac{1-F\left(\beta_{1}\right)}{F^{\prime}\left(\beta_{1}\right)}=\left[\theta_{3}+b_{B}\left(m_{3}\right)\right]-\left[\theta_{1}+b_{B}\left(m_{1}\right)\right]+\beta_{1}
$$




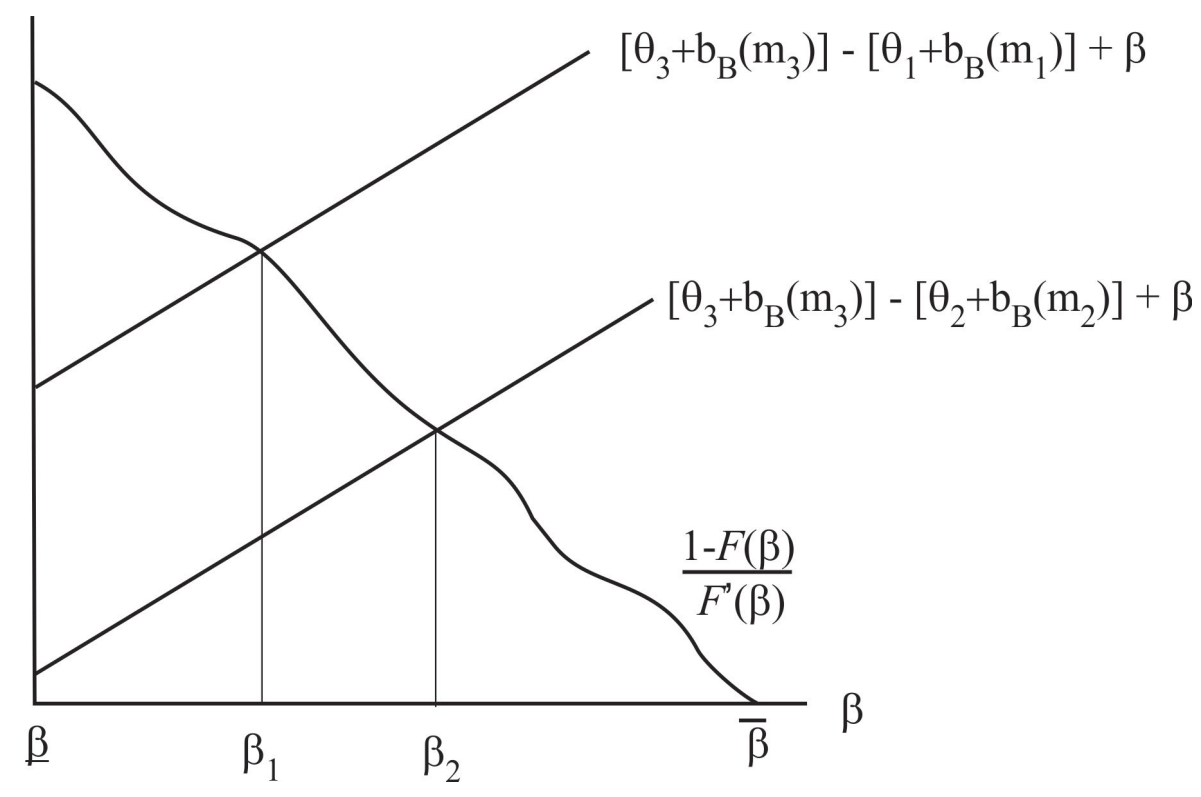

Figure 1: Interior Solution

and

$$
\frac{1-F\left(\beta_{2}\right)}{F^{\prime}\left(\beta_{2}\right)}=\left[\theta_{3}+b_{B}\left(m_{3}\right)\right]-\left[\theta_{2}+b_{B}\left(m_{2}\right)\right]+\beta_{2} .
$$

Figure 1 plots the right-hand sides and left-hand sides of (9) and (10) as functions of the political benefits $\beta \in[\beta, \bar{\beta}]$ on the horizontal axis. The lefthand side of the equilibrium conditions above is non-increasing due to the monontone-hazard-rate condition. For $\beta=\bar{\beta}$, the left-hand side becomes zero. The right-hand sides are straight lines with slope 1 . Note that $\left[\theta_{1}+\right.$ $\left.b_{B}\left(m_{1}\right)\right]<\left[\theta_{2}+b_{B}\left(m_{2}\right)\right]$, which implies that $\beta_{2}>\beta_{1}$. This, in turn, implies that the probability for success in case $k=1,1-F\left(\beta_{1}\right)$, is higher than in case $k=2$, which is $1-F\left(\beta_{2}\right)$. Putting it differently, the chances that the offer is accepted is smaller if a CDM is generally available than without it.

Proposition 1 is the main result of the paper. If the incomplete information about the responder's attitude towards an agreement causes ultimatum bargaining to fail with some probability, this probability is higher if countries can rely on a CDM in the non-cooperative fallback. The intuition for this result is that the fallback position affects the offers. If there is no CDM in 
place in the non-cooperative outcome, the gains from successful negotiations are larger. Therefore, proposer $A$ is more cautious / less aggressive when making its take-it-or-leave-it offer to the responder $B$. Another interpretation brings the result in line with what is known in the context of industrial organization as the topsy-turvy-principle: cooperation works better if the non-cooperative outcome is worse.

Corner solutions So far we considered cases in which the optimal offer (both in the regime without and with a CDM in the non-cooperative fallback) is an 'interior solution' for $t_{1}$ and $t_{2}$ as solutions to (6) and (8), such that the corresponding critical levels $\beta_{1}\left(t_{1}\right) \in(\beta, \bar{\beta})$ and $\beta_{2}\left(t_{2}\right) \in(\beta, \bar{\beta})$. This means that, for the optimal transfer offers chosen, the responder $B$ has an acceptance probability that is strictly positive, but smaller than 1. An interior solution cannot be taken for granted. This is evident from Figure 1. An interior solution requires that the upward sloping curves $\left[\theta_{3}+b_{B}\left(m_{3}\right)\right]-\left[\left(\theta_{k}+b_{B}\left(m_{k}\right)\right]+\beta\right.$ intersect $(1-F(\beta)) / F^{\prime}(\beta)$ for a value of $\beta \in(\beta, \bar{\beta})$. In this section, we want to demonstrate that our main result also holds qualitatively for corner solutions.

The optimal choice of the proposer may lead to a 'corner solution', of which two types can emerge. The first, to which we refer as the 'noncooperative corner solution' in what follows, is simply the non-cooperative outcome, which can be reached by a transfer demand $t_{k}$ with $\beta_{k}\left(t_{k}\right)>\bar{\beta}$, that is so high that $B$ would never accept it for any $\beta \in[\underline{\beta}, \bar{\beta}]$. This leads to an equilibrium payoff for $A$ of $\theta_{k}$ for $k \in\{1,2\}$. The second, to which we refer to as the 'cooperative corner solution', is a choice of $t_{k}$ with $\beta_{k}\left(t_{k}\right)<\underline{\beta}$, that is so low that $A$ can be sure that $B$ accepts. Among the $t_{k}$ with this property, $A$ prefers to ask for the largest $t_{k}$. For $k=1$, i.e., if a CDM is not available in the non-cooperative fallback, this largest, always accepted $t_{k}$ is determined by

$$
\underline{t}_{1}=b_{B}\left(m_{3}\right)-b_{B}\left(m_{1}\right)+\underline{\beta} .
$$

Similarly, if a CDM is available in the fallback, then the largest transfer demand that is accepted for all types of responders $B$ is determined by

$$
\underline{t}_{2}=b_{B}\left(m_{3}\right)-b_{B}\left(m_{2}\right)+\underline{\beta} .
$$

The proposer $A$ 's payoff in this corner solution is

$$
\theta_{k}+b_{B}\left(m_{3}\right)-b_{B}\left(m_{k}\right)+\underline{\beta}, \text { for } k=1,2 .
$$

Whether a corner solution emerges and, if so, which one, depends on the range of political benefits $\beta \in[\underline{\beta}, \bar{\beta}]$ and on the function $F(\beta)$. A look at 
the first derivative of $A$ 's expected profit yields insights into the conditions for the corner solutions. This derivative is

$$
\frac{\partial E \pi_{A, k}}{\partial t}=\left[1-F\left(\beta_{k}(t)\right)\right]-\left(\theta_{3}-\theta_{k}+t\right) \cdot F^{\prime}\left(\beta_{k}(t)\right)
$$

The first term $(1-F)$ is $A$ 's expected benefit from a marginal increase of the transfer $t$; $A$ receives the additional transfer with probability $1-F$. The second term is the expected cost of a marginal increase in the transfer; the probability of receiving the benefit from successful bargaining $\left(\theta_{3}-\right.$ $\left.\theta_{2}+t\right)$ falls by $F^{\prime}$ when the transfer is marginally increased. It takes into consideration that $\partial \beta_{k} / \partial t=1$ for the range $\beta_{k}(t) \in(\underline{\beta}, \bar{\beta})$ and $\partial \beta_{k} / \partial t=0$ for the range $\beta_{k}(t) \notin[\underline{\beta}, \bar{\beta}]$.

The non-cooperative corner solution emerges if $\theta_{k}$ with probability 1 yields a higher payoff for $A$ than any offer $t_{k}$ that would be accepted with positive probability. This requires in particular that $\frac{\partial E \pi_{A, k}}{\partial t}>0$ at the $t_{k}$ that makes $B$ just indifferent between acceptance and rejection if $\beta=\bar{\beta}$. I.e., it requires

$$
\frac{\partial E \pi_{A, k}}{\partial t}=[1-F(\bar{\beta})]-\left(\left[\theta_{3}+b_{B}\left(m_{3}\right)\right]-\left[\theta_{k}+b_{B}\left(m_{k}\right)\right]+\bar{\beta}\right) \cdot F^{\prime}(\bar{\beta})>0
$$

This necessary condition is also sufficient for ruling out that an interior solution with a threshold $\beta \in(\beta, \bar{\beta})$ is optimal. To show this, consider transfer demands that yield thresholds $\beta<\bar{\beta}$. Compared to $\beta=\bar{\beta}$, we see that $[1-F(\beta)]$ will become positive. Further, $\left(\left[\theta_{3}+b_{B}\left(m_{3}\right)\right]-\left[\theta_{k}+\right.\right.$ $\left.\left.b_{B}\left(m_{k}\right)\right]+\beta\right)$ is monotonically increasing in $\beta$. Accordingly, $\frac{\partial E \pi_{A, k}}{\partial t} \geq 0$ in the whole range $\beta \in[\beta, \bar{\beta}]$. We conclude: as $F(\bar{\beta})=1$, the first corner solution will be chosen if the gain from successful negotiations is outweighed by the political disutility of the responder with the highest valuation: $\left[\theta_{3}+\right.$ $\left.b_{B}\left(m_{3}\right)\right]-\left[\theta_{k}+b_{B}\left(m_{k}\right)\right]<-\bar{\beta} \cdot{ }^{8}$

Figure 2 illustrates this outcome. The downward-sloping curve meets the horizontal axis at $\beta=\bar{\beta}$. If the upward sloping curves end beneath the horizontal axis, country $A$ will choose the non-cooperative solution. Comparing the outcomes without $(k=1)$ and with $(k=2)$ a CDM in the fallback position confirms our earlier result for interior solutions. The chances for successful negotiations cannot be improved by allowing domestic firms to abate abroad. An increase in the benefit $\beta$ from successful negotiations shifts both straight lines upwards. As the upward-sloping line without CDM

\footnotetext{
${ }^{8}$ Note that $[1-F(\bar{\beta})]=0$. Further, $\left(\left[\theta_{3}+b_{B}\left(m_{3}\right)\right]-\left[\theta_{k}+b_{B}\left(m_{k}\right)\right]+\bar{\beta}\right)>0$ and $F^{\prime}(\beta)>0$.
} 


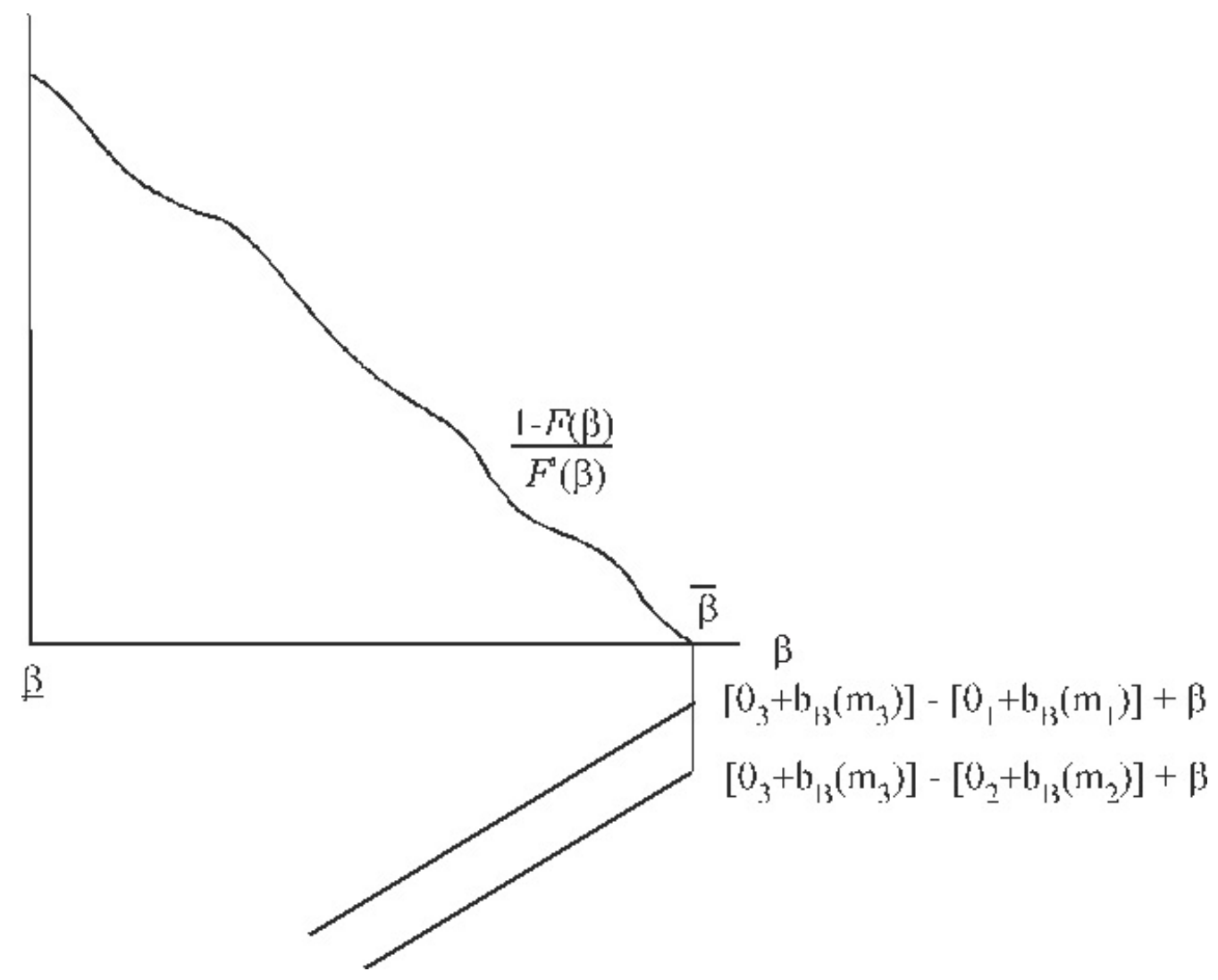

Figure 2: Non-cooperative Corner Solution

$(k=1)$ lies above the line with CDM $(k=2)$, the chances that a country leaves the non-cooperative solution must be higher when the country does not allow to abate abroad in the fallback position.

A similar line of reasoning can be applied for the other corner solution where country $A$ makes such a cautious proposal that country $B$ will accept the offer for all $\beta$-values. A necessary condition for such a cautious proposal to be optimal for $A$ is

$$
\frac{\partial E \pi_{A, k}}{\partial t}=[1-F(\underline{\beta})]-\left(\left[\theta_{3}+b_{B}\left(m_{3}\right)\right]-\left[\theta_{k}+b_{B}\left(m_{k}\right)\right]+\underline{\beta}\right) \cdot F^{\prime}(\underline{\beta})<0 .
$$

The condition describes that a marginal increase in the transfer at $\underline{t}_{k}$ does not increase $A$ 's expected payoff. This necessary condition is also sufficient. 


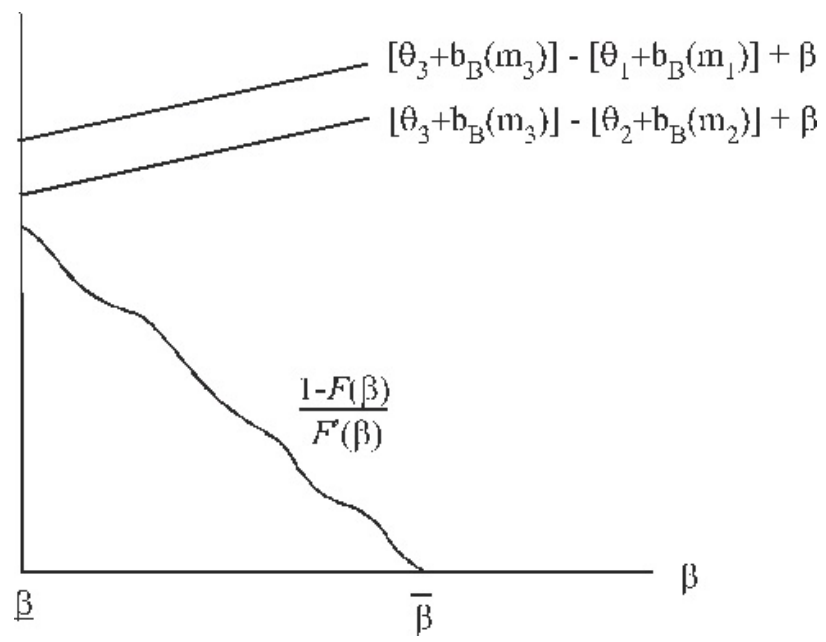

Figure 3: Fully Cooperative Corner Solution

If (15) holds at $t=\underline{t}_{k}$ then $\frac{\partial E \pi_{A, k}}{\partial t} \leq 0$ holds also for any higher $t$. To see this, consider the two additive terms in (15). One of these is $(1-F)$; it is positive and decreasing in $t$. The other term is $-\left(\left[\theta_{3}+b_{B}\left(m_{3}\right)\right]-\left[\theta_{k}+\right.\right.$ $\left.\left.b_{B}\left(m_{k}\right)\right]+\beta\right) \cdot F^{\prime}(\beta)$ and may decrease or increase in $t$. However, the sign of $\frac{\partial E \pi_{A, k}}{\partial t}$ at any $t$ for which $F^{\prime}>0$ is the same as the sign of

$$
\frac{[1-F(\beta)]}{F^{\prime}(\beta)}-\left(\left[\theta_{3}+b_{B}\left(m_{3}\right)\right]-\left[\theta_{k}+b_{B}\left(m_{k}\right)\right]+\beta\right) .
$$

Now we see that the first term is non-increasing in $\beta$ (hence, in $t$ ) due to Assumption $(\mathrm{H})$. The second term is negative and strictly decreasing in $\beta$. Accordingly, if (16) is non-positive at $\beta=\underline{\beta}$ then it never becomes positive for larger values of $\beta$.

The cooperative corner solution is illustrated in Figure 3. If the marginal benefit of an increase in $t$ beyond $\underline{t}_{k}$ is below the marginal cost, the proposer sets $\underline{t}_{k}$ and reaches an agreement with probability 1 . Again, as the upwardsloping line for $k=1$ lies above the line for $k=2$, it is more likely that the cautious solution is chosen when the CDM is not implemented in the fallback position. Put differently, if the distribution of political benefits becomes wider and $\beta$ falls, a country will leave its cautious corner solution earlier when the $\mathrm{CDM}$ is place compared to the case without CDM.

We summarize these findings in 
Proposition 2 For a given distribution $F(\beta)$ with $\beta \in[\beta, \bar{\beta}]$ the following holds: (i) If a cooperative corner solution applies for $k=\overline{2}$, then a cooperative corner solution also applies for $k=1$. (ii) If a non-cooperative corner solution applies for $k=1$, then a non-cooperative corner solution also applies for $k=2$.

Proof. By (15) the proposer in country $A$ prefers the cooperative corner solution if

$$
\frac{\partial E \pi_{A, k}}{\partial t}=[1-F(\underline{\beta})]-\left(\left[\theta_{3}+b_{B}\left(m_{3}\right)\right]-\left[\theta_{k}+b_{B}\left(m_{k}\right)\right]+\underline{\beta}\right) \cdot F^{\prime}(\underline{\beta})<0 .
$$

As $\theta_{2}+b_{B}\left(m_{2}\right)>\theta_{1}+b_{B}\left(m_{1}\right)$, for any given $\beta$ this condition is fulfilled for $k=2$ if it is fulfilled for $k=1$. This establishes (i). Similarly, for (ii), consider the condition (14) which is the sufficient for a non-cooperative corner solution:

$$
\frac{\partial E \pi_{A, k}}{\partial t}=[1-F(\bar{\beta})]-\left(\left[\theta_{3}+b_{B}\left(m_{3}\right)\right]-\left[\theta_{k}+b_{B}\left(m_{k}\right)\right]+\bar{\beta}\right) \cdot F^{\prime}(\bar{\beta})>0 .
$$

By $\theta_{2}+b_{B}\left(m_{2}\right)>\theta_{1}+b_{B}\left(m_{1}\right)$ this condition holds for $k=1$ if it holds for $k=2$.

Proposition 1 showed that reaching an agreement is more likely for $k=1$ than for $k=2$ if the proposer $A$ chooses to accept a positive probability smaller than 1 of bargaining failure for both $k=1$ and $k=2$. Proposition 2 broadens the scope of Proposition 1 with respect to corner solutions. According to Proposition 2, a non-cooperative corner solution prevails if there is no CDM availiable in the non-cooperative fallback only if such a corner solution also occurs if a CDM is available in the non-cooperative fallback. Also, if bargaining leads to a cooperative corner solution even if a CDM is available in the non-cooperative fall-back, this implies that bargaining with a fallback without an CDM also leads to a cooperative corner solution. Hence, availability of a CDM may drive the bargaining outcome into a non-cooperative corner solution. Non-availability of a CDM can drive the bargaining outcome into full cooperation, even if a cooperative corner solution does not emerge if a CDM is available in the non-cooperative fallback.

For some distributions $F(\beta)$, the equilibrium leads to the same (noncooperative or cooperative) corner solution for both cases, with and without a CDM in the fallback, as depicted in Figures 2 and 3. For some $F(\beta)$, however, it may happen that the equilibrium is the non-cooperative corner solution for one CDM regime and a cooperative corner solution for the other 


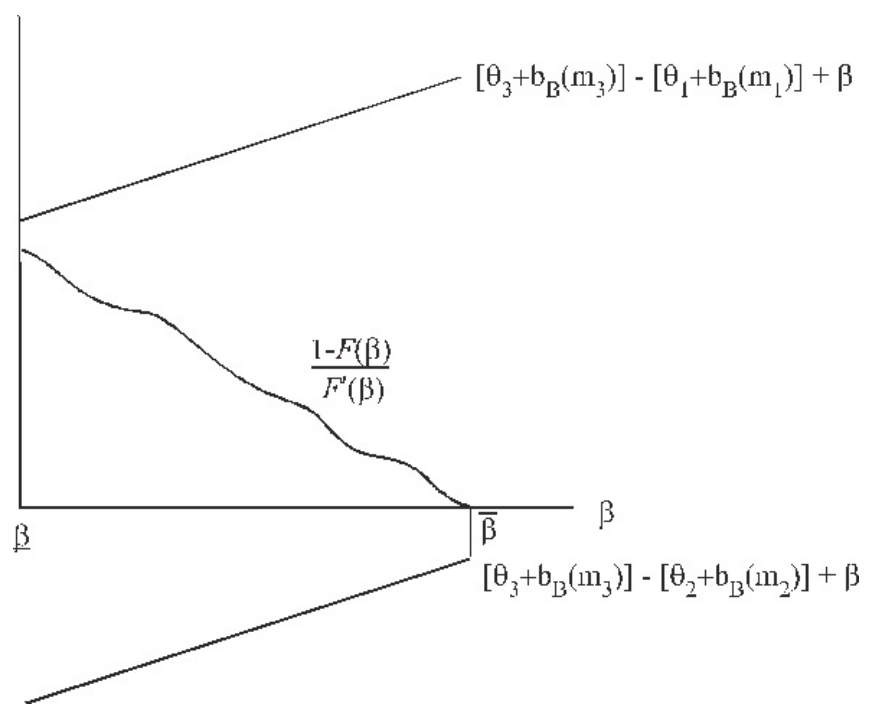

Figure 4: Both Corner Solutions

CDM regime. Such a case is illustrated in Figure 4. This figure also makes clear that, for such mixed cases, the cooperative corner solution emerges for the case $k=1$, in which a CDM is unavailable in the fallback, and the non-cooperative corner solution emerges for case $k=2$, in which the CDM is available in the fallback. The reverse mixed case is not feasible. Hence, availability of a CDM in the non-cooperative fallback may turn the bargaining equilibrium from reaching a cooperative negotiation outcome with certainty to one in which negotiations fail with probability 1.

There is a range in which an efficient agreement is reached if a CDM is not available in the fallback, but no such efficient agreement is reached if a CDM is available in the fallback. Proposition 2 reveals that a CDM is also detrimental for reaching a cooperative agreement if the equilibrium transfer demand does not lead to an interior outcome but to a corner solution for which cooperation is achieved with probability 1 or with probability 0 . For a set of given distributions $F(\beta)$, the range for which a cooperative outcome emerges is larger for $k=1$, i.e., for a case in which no CDM is available in the non-cooperative fallback. The intuition for the result is very similar to, and more straightforward than the intuition for Proposition 1: If a CDM is available in the non-cooperative outcome, this makes the non-cooperative outcome more attractive. The overall social surplus that can be achieved 
by a cooperative outcome is larger if a CDM is not available in the noncooperative outcome.

\section{Conclusions}

Our model exploits a simple mechanism to illustrate the feedback effects of a CDM on international environmental agreements. The CDM seems purely beneficial in the first place as it allows for additional abatement at low costs. Problems that may occur with a CDM in practical applications, such as the problem whether a CDM provides additionality, are absent in our framework. By definition all abatement units are additional here. Nevertheless, the implementation of a CDM has a servere drawback. It reduces the chances for successful international environmental treaties. A CDM that is in place before the international bargaining starts reduces the gains from successful negotiations. This in turn leads to more aggressive bargaining and, therefore, higher chances of failed negotiations.

Within the simple context of ultimatum bargaining our analysis highlights a result which may prove to be robust: making the outcome of negotiation failure in climate negotiations more attractive may reduce the efficiency of these negotiations. How the availability of a CDM affects efficiency in the equilibrium could be studied if a more differentiated picture of CDMs is drawn, or if contract negotiations take more sophisticated formats. These could include iterated bargaining or bargaining with alternating offers under two-sided incomplete information or negotiations that allow the proposer to offer a more sophisticated menu of contracts.

\section{References}

[1] Admati, A.R. and M. Perry, 1987, Strategic Delay in Bargaining, Review of Economic Studies 54, 345-364.

[2] Aresin, S., 2013, Cross Border Abatement and its Welfare Effects, Working Paper of the Max Planck Institute for Tax Law and Public Finance, 2013-04, Available at SSRN: http://ssrn.com/abstract=2307821 or http://dx.doi.org/10.2139/ssrn.2307821.

[3] Barrett, S., 1998, On the Theory and Diplomacy of Environmental Treaty-making, Environmental Resource Economics 11(3-4), 317-333. 
[4] Barrett, S., 2001, International Cooperation for Sale, European Economic Review 45, 1835-1850.

[5] Beccherle, J. and J. Tirole, 2011, Regional Initiatives and the Cost of Delaying Binding Climate Change Agreements, Journal of Public Economics 95, 1339-1348.

[6] Buchholz, W. and K.A. Konrad, 1994, Global Environmental Problems and the Strategic Choice of Technology, Journal of Economics 60, 299321.

[7] Carraro, C. and D. Siniscalco,1993, Strategies for the International Protection of the Environment, Journal of Public Economics 52, 309-328.

[8] Chatterjee, K., and W. Samuelson, 1983, Bargaining under Incomplete Information, Operations Research 31(5), 835-851.

[9] Harstad, B., 2007, Harmonization and Side Payments in Political Cooperation, American Economic Review 97(3), 871-889.

[10] Helm, C., and F. Wirl, 2014, The Principal-agent Model with Multilateral Externalities: An Application to Climate Agreements, Journal of Environmental Economics and Management 67(2), 141-154.

[11] Hoel, M., 1991, Global Environmental Problems - The Effects of Unilateral Actions Taken by One Country, Journal of Environmental Economics and Management 20(1), 55-70.

[12] Hoel, M. and K. Schneider, 1997, Incentives to Participate in International Environmental Agreements, Environmental and Resource Economics $9,153-170$.

[13] Konrad, K.A. and M. Thum, 2014, Climate Policy Negotiations with Incomplete Information, Economica 81, 244-256.

[14] Martimort, D. and W. Sand-Zantman, 2013, Solving the Global Warming Problem: Beyond Markets, Simple Mechanisms May Help! Canadian Journal of Economics 46(2), 361-378.

[15] Myerson, R.B., and M.A. Satterthwaite, 1983, Efficient Mechanisms for Bilateral Trading, Journal of Economic Theory 29(2), 265-281. 